Bangladesh Journal of Bioethics 2014; 5(3):1-5

\title{
Practice of Informed Consent, Confidentiality and Privacy by Physicians at a Tertiary Care Teaching Hospital
}

\author{
Zoheb Rafique ${ }^{1}$, Urooj Bhatti ${ }^{2}$
}

1 Lecturer, Department of Biochemistry, Liaquat University of Medical \& Health Sciences (LUMHS), Jamshoro, Pakistan

2 Lecturer, Department of Physiology, Liaquat University of Medical \& Health Sciences (LUMHS), Jamshoro, Pakistan

Email: $\underline{\text { dr zohaib@hotmail.com }}$

\begin{abstract}
Objective: The aim of this paper was to assess the practice of medical ethics by the physicians at a public sector hospital in jamshoro Sindh. Material and methods: This survey was conducted at four medical units of tertiary care hospital at jamshoro in the month of august 2014. Participants were randomly selected from patients aged over 18 years. A structured questionnaire was designed and the participants were asked about their demographic profile and their physician's practice regarding informed consent, maintaining confidentiality, privacy and other treatment formalities. Written consent was taken from all the participants before interview. Results: A total of 100 patients were randomly selected for this study. The majority of patients reported that informed consent was taken from them. The patients also responded that privacy and confidentiality is maintained during their treatment. However, many patients agreed that they were not properly informed about the laboratory findings, role of proposed drugs and also side effects of drugs. Conclusion: There is marked improvement in the practice of medical ethics by physicians of this tertiary care hospital. However, awareness workshops should be conducted to update and improve the knowledge of medical ethics among physicians. This will surely help them translate the knowledge into practice.
\end{abstract}

Key words: informed consent, confidentiality, privacy

Introduction: In the rapidly changing system of health care, many different factors have affected and changed the perception about how health care is practiced today. The rights of patient are also affected. Patient rights have now become the center of attention in practice of medicine. Today, concerns about patients' choice and the respect for their preferences, values and the access to medical care are getting more complex. The Patients' expectations are becoming higher and now they always want every thing best. They want active participation in decision making and proposed treatments or procedures and the other alternatives available ${ }^{1}$. Information regarding designed surgical or medical intervention should be presented to patients before starting any procedure or treatment. In addition to this, the risks of treatment 
as well as benefits should also be explained to the patient, so that patient can make a decision regarding what he/she demands. It is recognized as an essential measure, to ensure the preservation of patient rights. The requirement for obtaining informed consent is established in every decision making situation in clinical practice. Fully informed patient is able to participate in decisions regarding his/her health care ${ }^{2}$. Along with informed consent, respect for confidentiality and privacy is also included in responsibility of doctors/physicians throughout history. Very recently though attention has been centered on these principles with the formal introduction in modern codes of medical ethics. The concepts of confidentiality and privacy are closely related. Confidentiality is a narrow term referring to informational privacy and also to the responsibility not to disclose any information about patients without prior permission from the patient. Privacy is a broad term including informational privacy, physical privacy, protection of the personal identity and ability to make informed choices without interference. Confidentiality and Privacy are basic rights of patients and they also serve to nurture a frank, trustful and open relationship with doctor/physician, thus improving patient care $^{3}$. Numerous studies have shown that doctors in routine medical practice don't consider it necessary to take proper consent after providing the patients with thorough information. Similarly despite the fact that respect for confidentiality and privacy has been a responsibility of physicians/doctors throughout history, it is noted that doctors often neglect the aspect of medical ethics. However, despite the fact that regulatory body for medicine in our country, the Pakistan Medical and Dental Council commonly known as (PMDC), has formulated code of ethics for doctors, no major steps have been taken in order to ensure its implementation. Recently, there has been a significant change in the thinking of the patients, mainly because of greater influence of ICT (Information Communication Technology, primarily of electronic media and the internet). Thus, due to the increasing awareness, now patients demand full information about treatment options, treatment plans and also possible complications and these aspects were not practiced in the past ${ }^{4}$. In this paper the patient's perceptions regarding practice of physicians in a tertiary care teaching hospital was discussed.

Material and Methods: To assess the perception of patients in the medicine wards regarding practice of medical ethics by the physicians, a survey was conducted at Liaquat University of Medical and Health Sciences (LUMHS) Hospital Jamshoro in the month of august 2014. A structured questionnaire was designed and the patients were then interviewed. A total of 100 patients were randomly selected in four medical units. The patients were taken above the age of 18 years, so that they can understand and give consent themselves. Written consent was taken at the time of interview. The questionnaire consisted of eight (08) questions and it took 15 minutes to complete the interview and fill the questionnaire.

Results: A total number of 100 patients participated in this study. Our study population included 59 male patients and 41 female patients. The demographic data of patients is shown in Table 1 and Table 2 shows the patient's responses to the questionnaire. 
Table: 1 Demographic Profile

\begin{tabular}{|cc|}
\hline Sex & No of Patients \\
\hline Male & 59 \\
\hline Female & 41 \\
\hline
\end{tabular}

Table: 2 Shows the perception of patients regarding physicians practices

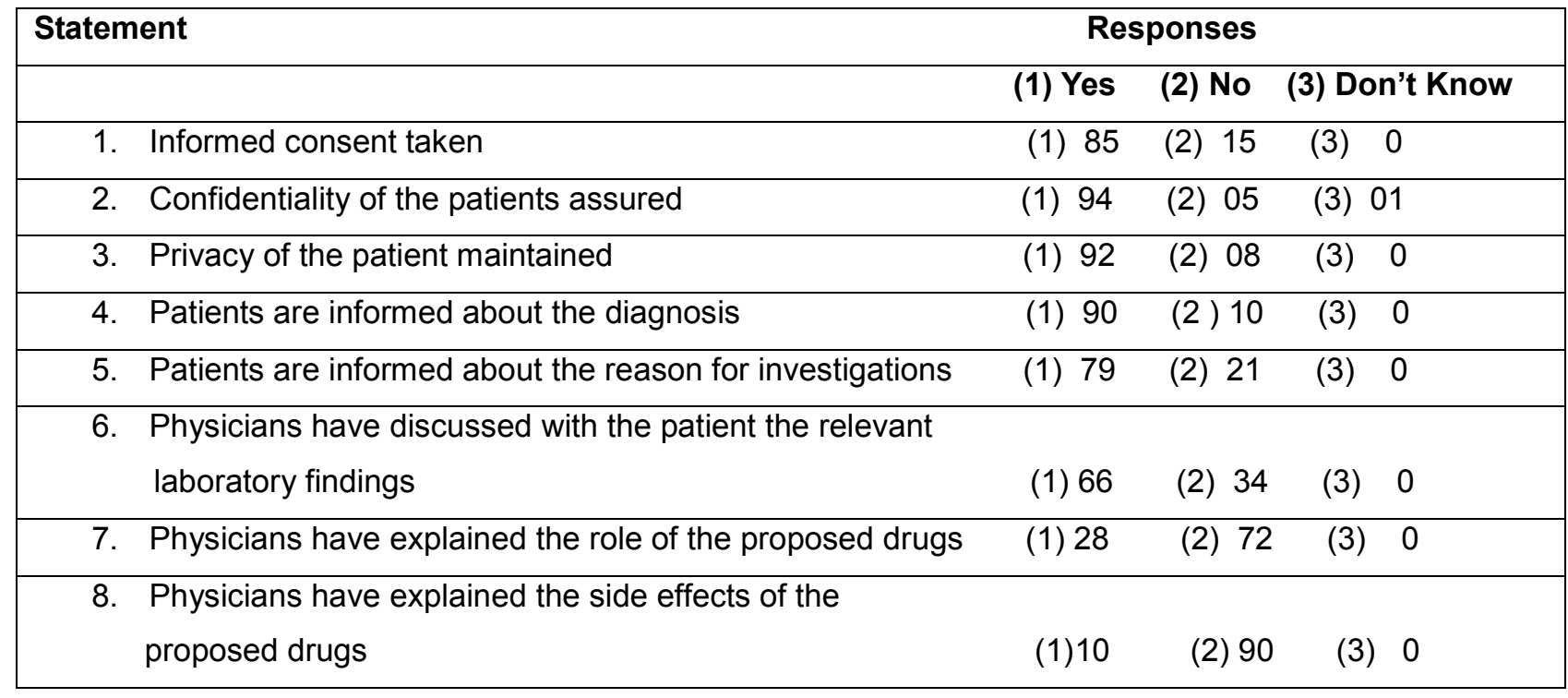

Discussion: This study was designed to assess the practice of informed consent, confidentiality, privacy and other treatment modalities by physicians at a tertiary care hospital. In our study $85 \%$ of the patients were of the view that informed consent was taken from them. We asked about taking informed consent during general physical examination, history taking and routine procedures. This percentage is very good because of the reason that we have teaching hospital and now bioethics is being taught at undergraduate as well as postgraduate level, so physicians are familiar with informed consent, its components and requirements. We also looked at physician's practice of maintaining and assuring privacy and confidentiality. In our study we found that $94 \%$ of the patient agreed that confidentiality was assured and maintained during their treatment and only $6 \%$ of the patient either disagreed or not sure about their confidentiality. When we asked about the maintenance of privacy, $92 \%$ of the patients were of the view that privacy was maintained during their examination, treatment and routine procedures and only $8 \%$ of the patients negated this statement. Our study data is better then other studies conducted in Pakistan indicting that physicians are aware of the actual practice of medical ethics. In one question we asked the patients about the information regarding the diagnosis, 90 percent of the patient agreed that they were informed about the diagnosis or the disease they are suffering, while $10 \%$ patients disagreed and were not told about their diagnosis. $79 \%$ of the patients were of the view that they were informed about the 
reason for investigation/laboratory reports and $21 \%$ disagreed and were having view that they were not informed about the reason for having different laboratory reports, blood tests, urine tests and other investigations etc. When asked about the discussion of relevant laboratory findings, only $66 \%$ of the patient agreed and $34 \%$ of the patients disagreed with the statement. In our last two questions we asked the patients about the role of the drugs given to them by the physicians and also the side effects of those drugs. We found that only $28 \%$ of the patients agreed that they were explained in detail the role of the drugs given to them, means why that particular drug is given and for how much time and at what time the patents have to take those drugs and 72 percent of the patient disagreed. Only $10 \%$ of the patient agreed that they were explained the side effects of the drugs which they were taking and 90 percent disagreed and told us that they were not briefed about the side effects of the treatment they took. When we discussed these issues in detail with some patients and also with some residents/physicians working in medicine wards, we found some interesting answers. Doctors say that majority of patients are illiterate, so it is of no use to tell them about the laboratory findings and drug side effects in detail. Some physicians were of the view that patients and their relatives are only interested to see patient get well soon and they are not interested how and why. Patients were having view that doctors are over burdened. Some patients say doctors are less interested and some physician's say that patients are not educated much and they don't understand even the basic things some time, so therefore they don't discuss things in detail. Residents say they have less house officers and senior doctors and consultants don't give much time and some patients say that doctors are sincere with their duty. Informed consent enjoys unassailable position in both research and clinical situations as safeguard of patients' rights. The involvement of the patient in decision making process is much easier when there is direct link/communication with the individual. The Pakistani milieu carries challenges to this process because important decision making is very often done by the family members or in some cases it is left entirely to the attending physician. This also raises some ethical dilemmas for the physicians who may not feel comfortable with the communication which excludes the patient or in accepting the paternalistic decision making role ${ }^{5}$. This study has one potential limitation that this study was conducted in one single hospital and its results cannot be generalized for all government/public sector units/hospitals in Pakistan.

Conclusion: The results of our study suggest that physicians at teaching hospitals may have improved their practice of medical ethics. However, due to the fact that data was collected only at one tertiary care/teaching hospital, we can't comment on ethical practices of the physicians from private healthcare sector and other public sector hospitals. Nonetheless, to encourage the ethical practice, steps should be taken in highlighting the importance of basic principles of medical ethics: informed consent, confidentiality and privacy. Furthermore, awareness campaign should be conducted for the physicians to improve their knowledge of the ethical principles. 


\section{References:}

1. Farida M, Habib and Hind Sulaiman Al $\square$ Siber. Assessment of Awareness and Source of Information of Patients' Rights: a Cross $\square$ sectional Survey in Riyadh Saudi Arabia. American Journal of Research Communication. 2013; 1(2):1-9.

2. Faisal Ghani Siddiqui, Jan Mohammad Shaikh, Mohammad Munir Memon. An Audit of Informed Consent in Surgical Patients at a University Hospital. J Ayub Med Coll Abbottabad. 2010; 22(1).

3. Ayesha Humayun Et Al. Patients' perception and actual practice of informed consent, privacy and confidentiality in general medical outpatient departments of two tertiary care hospitals of Lahore. BMC Medical Ethics. 2008; 9:14.

4. Waris Qidwai Et Al. Informed consent, privacy and confidentiality practiced by doctors of a tertiary care hospital in a developing country. Indian Journal of Medical Ethics. 2013; 9(1).

5. A M Jafarey, A Farooqui. Informed consent in the Pakistani milieu: the physician's perspective. J Med Ethics. 2005; 31:93-96.

Conflict of Interest: There is no conflict of interest. 